\section{Human dentin phosphophoryn nucleotide and amino acid sequence}

Gu K, * Chang SR, ${ }^{*}$ Slaven MS, Clarkson BH, Rutherford RB, Ritchie HH: Human dentin phosphophoryn nucleotide and amino acid sequence. Eur J Oral Sci 1998; 106: 1043-1047. (C) Eur J Oral Sci, 1998

Dentin sialoprotein (DSP) and phosphophoryns (DPP) are major dentin-specific non-collagenous proteins and are synthesized by odontoblasts. DPP are extremely acidic, rich in aspartic acid and serine, possess a high affinity for calcium and collagen, and are believed to function in dentin mineralization. Whereas DSP and DPP are the products of a single gene in mouse and rat, an analogous human gene has not been described. Using RT-PCR based cloning strategies, we have cloned human DPP cDNA from immature molar root total RNA. The open reading frame of this human DPP cDNA comprises $2364 \mathrm{bp}$ encoding 788 amino acids rich in serine (58\%), aspartic acid $(26 \%)$ and asparagine (9\%). These are mostly arranged as (DSS) $(n=1-16)$, DS and NSS motifs. The N-terminal sequence (DDP) matches that obtained from human DPP extracted from the roots of immature teeth. The core protein of this human DPP was calculated to have a molecular weight of $76,906 \mathrm{Da}$ and a net charge of -206 with an isoelectric point of 2.65 . Of the serine residues, $53 \%$ can potentially be phosphorylated by casein kinases I and II. Thus, this newly cloned human cDNA, which encodes a protein with characteristics similar to rat and mouse DPP, is identified as a human DPP.
Keni Gu, Syweren R. Chang, Matt S. Slaven, Brian H. Clarkson, R. Bruce Rutherford, Helena H. Ritchie

Department of Cariology, Restorative Sciences and Endodontics, School of Dentistry, University of Michigan, Ann Arbor, MI, USA
Bruce Rutherford, Department of Cariology, Restorative Sciences and Endodontics, School of Dentistry, University of Michigan, Ann Arbor, MI 48109-1078, USA

Telefax: +1-734-9361597

E-mail: rbruth@umich.edu

Key words: human dentin phosphoprotein; nucleotide sequence; amino acid sequence

Accepted for publication September 1998
Mammalian dentin matrices contain collagen (principally type I) and several non-collagenous proteins (NCP). Principal among these are several isoforms of phosphoprotein (DPP) and dentin sialoprotein (DSP) comprising approximately $50 \%$ and $5 \%$ of dentin NCP, respectively (1-3). Interestingly, these two molecules which are present in dentin as distinct proteins are encoded by a single gene in both rat (3-5) and mouse (6). This gene, referred to as dentinsialophosphoprotein (DSPP (6)), comprises at least 5 exons in both mouse (7) and rat (3) genomes. Exons 1-4 encode DSP, while exon 5 encodes the mouse/rat DSP C-terminus as well as the entire mouse DPP. The structure and function of these distinct and unique dentin proteins, which are proposed to play crucial roles in tooth morpho-

\footnotetext{
* These authors contributed equally to this project.
}

genesis and the mineralization of dentin matrices, have recently been reviewed $(1,3,8)$.

Limited data are available on human DSP and DPP. Human immature roots contain a highly phosphorylated phosphoprotein with an apparent molecular weight of $140 \mathrm{kDa}$ by SDS-PAGE (9); this cannot be detected in dentin from fully mature (closed root apices) human permanent teeth (10). The amino terminal sequence of this phosphoprotein is aspartic acid (D) -aspartic acid (D) -proline (P) (9). Additional sequencing has not been possible due to the instability of the phosphorylated peptide chain (Crossley et al. report 1089 at the 1996 IADR meeting). DSPP has been localized to human chromosome $4 \mathrm{q}$ within the dentinogenesis imperfecta type II (DI-II) locus (11). Additionally DPP levels are reduced in individuals with DI-II $(12,13)$. Taken together such findings support a role for DPP in dentin mineralization in 


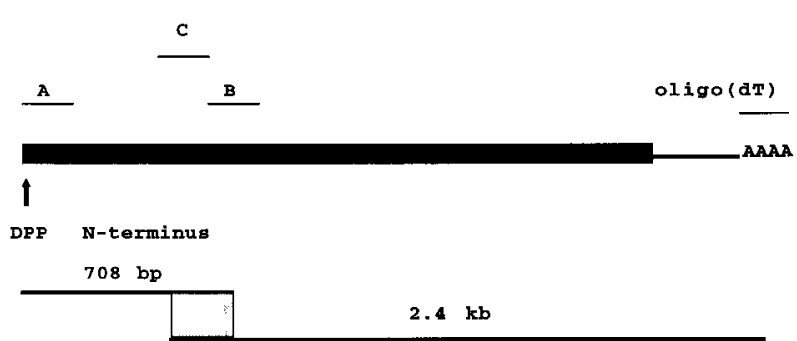

Fig. 1. Cloning of human DPP cDNA from immature molar tooth root total RNA by RT-PCR. A 708 bp PCR product was generated from human odontoblast and dental pulp cDNA pool with forward primer A (5'caaggagacgatcccaac $\left.3^{\prime}\right)$ corresponding to the rat DSP link sequence QG and N-terminal DPP sequence DDPN and reverse primer B (5'gctactgtcgctactgtc $\left.3^{\prime}\right)$ corresponding to rat DSSDSS (5). A second PCR fragment with a size of $2.4 \mathrm{~kb}$ was generated by $3^{\prime} \mathrm{RACE}$ with an oligo(dT) primer $\left(5^{\prime}\right.$ ggccacgcgtcgactagtact $\left.{ }_{17} 3^{\prime}\right)$, primer $\mathrm{C}$ (5'acagcagcaaatcagagagc $\left.3^{\prime}\right)$ derived from human DPP and an abridged universal amplification reverse primer. The $708 \mathrm{bp}$ and $2.4 \mathrm{~kb}$ PCR fragments were subcloned into TA vector pCR ${ }^{\text {II-TOPO }}$ and DNA sequences were determined. The gray zone represents the $215 \mathrm{bp}$ overlapped region of these two cDNA clones, which was confirmed by aligning several RTPCR-derived clones which used primers based on the human sequence.

human teeth. Therefore, it is important to clone and sequence human DPP to further our understanding of its role in human dentinogenesis, dentin repair and remineralization, and in abnormalities such as dentinogenesis imperfecta type II.

Using reverse transcriptase-polymerase chain reaction- (RT-PCR) based cloning strategies, we have cloned human DPP cDNA and determined its sequence. Here we report the first nucleotide and deduced amino acid sequence for the human DPP.

\section{Materials and methods}

\section{Isolation of total RNA}

Root tips were removed from freshly extracted human immature teeth (apices approximately $1 \mathrm{~mm}$ or greater diameter) and crushed into small shards. Approximately $200 \mathrm{mg}$ of dentin shards were mixed with $1 \mathrm{ml}$ of RNA STAT-60 (TELTEST B, Friendswood, TX, USA) and mechanically homogenized. The homogenate was mixed with 0.2 volume of chloroform followed by phase separation. The RNA from the aqueous phase was precipitated with an equal volume of isopropanol at room temperature, centrifuged at $11,000 \times g$ at $4{ }^{\circ} \mathrm{C}$ for $15 \mathrm{~min}$ and finally dissolved in DEPCtreated water and quantitated by UV absorbance spectrometry.

\section{Primer design and RT-PCR}

Human odontoblast and dental pulp cDNA pool was synthesized from total RNA obtained from the immature molar teeth root tips using random hexamers and reverse transcriptase according to the manufacturer's protocol (Perkin Elmer Cetus, Foster City, CA, USA). PCR was then performed using forward primer A (5'caaggagacgatcccaac $\left.3^{\prime}\right)$, and reverse primer B (5'gctactgtcgctactgtc $3^{\prime} ;$ Fig. 1$)$, based upon rat DPP (5) with the following program: $94^{\circ} \mathrm{C}$ for $30 \mathrm{~s}, 51^{\circ} \mathrm{C}$ for $15 \mathrm{~s}$ and $72^{\circ} \mathrm{C}$ for 2 min for 35 cycles. To obtain a cDNA sequence containing the polyA region, 3'RACE (rapid amplification of cDNA ends; Gibco-BRL) was performed with AMV reverse transcriptase and an oligo(dT)-adaptor primer (5'ggccacgcgtcgactag$\operatorname{tact}_{17} 3^{\prime}$; Gibco BRL,) to synthesize a cDNA pool, and followed by PCR with a forward primer C (5'acagcagcaaatcagagagc3'; Fig. 1) and an abridged universal amplification reverse primer (auap) with the following program: $94^{\circ} \mathrm{C}$ for $30 \mathrm{~s}$, $54^{\circ} \mathrm{C}$ for $15 \mathrm{~s}$ and $72^{\circ} \mathrm{C}$ for $3 \mathrm{~min}$ for 35 cycles. The primer pairs $\mathrm{A} / \mathrm{B}$ and the upper primer $\mathrm{C}$ (Fig. 1) for $3^{\prime}$ RACE were prepared using Oligo ${ }^{\mathrm{TM}}$ (Primer Analysis Software v.4, Wojciech Rychlik, Cincinnati, OH, USA).

\section{Sequencing}

An aliquot of the PCR mixture was resolved by agarose gel electrophoresis, and the appropriately sized bands were cut from the gel. The DNA was purified (Geneclean Kit; Bio 101, La Jolla, CA, USA), subcloned into the vector $\mathrm{pCR}^{\mathrm{II}-\mathrm{TOPO} T M}$ and transformed into One Shot ${ }^{\mathrm{TM}}$ competent cells (TA Cloning Kit; Invitrogen, San Diego, CA, USA). The plasmid was isolated by the Qiagen (Chatsworth, CA, USA) mini-prep protocol and sequenced by a fluorescence-labeling technique (PRISM $^{\mathrm{TM}}$ Ready Reaction DyeDeoxy ${ }^{\mathrm{TM}}$ Terminator Cycle Sequencing Kit; Perkin Elmer-Applied Biosystems, Foster City, CA, USA).

\section{Results and discussion}

An RT-PCR based cloning strategy was selected to clone human DPP from total RNA prepared from immature root tips. Root tips were selected because this tissue provides a good natural source of undegraded human DPP (9). Using primer pair A/B (Fig. 1), a 708 bp PCR product was generated, subcloned and sequenced. The portions of this sequence that contained the rat primer sequences (primers A/B) were corrected by the construction of human gene specific primers delineating regions, which spanned the primers. This corrected $708 \mathrm{bp}$ fragment contained a deduced N-terminal sequence (Fig. 2) which is identical to both the rat DPP N-terminal sequence 


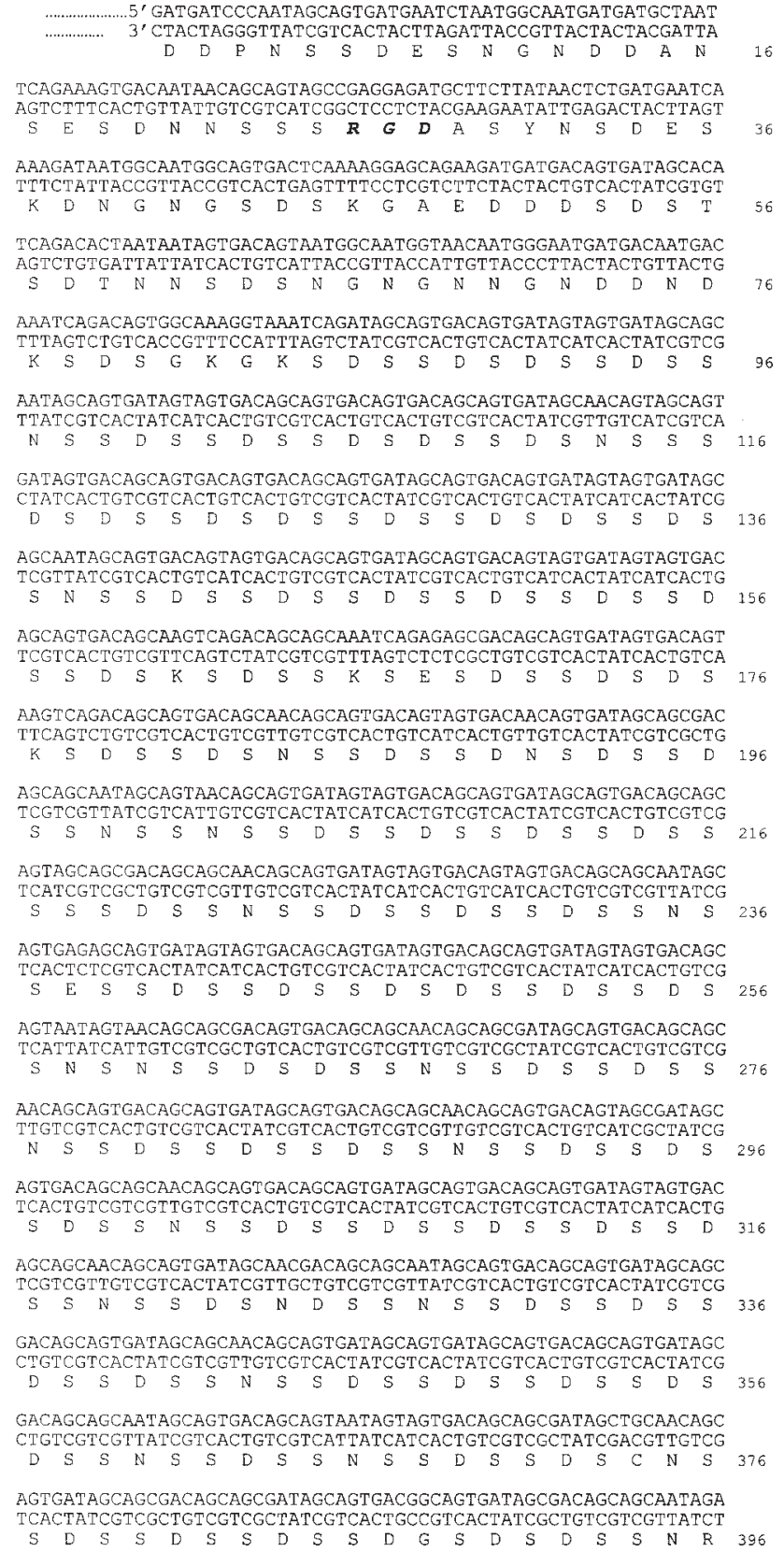

AGTGACAGTAGTAATAGTAGTGACAGCAGCGATAGCAGTGACAGCAGCAACAGCAGTGAC TCACTGTCATCATTATCATCACTGTCGTCGCTATCGTCACTGTCGTCGTTGTCGTCACTG AGCAGTGATAGCAGTGACAGCAACGAAAGCAGCAATAGCAGTGACAGCAGTGATAGCAGC TCGTCACTATCGTCACT'STCG'TI'GCTTICGTCGT'TATCGTCACTGTCGTCACT'ATCGTCG $\begin{array}{llllllllllllllllllll}S & \text { S } & S & S & D & S & N & E & S & S & N & S & S & D & S & S & D & S & S & 436\end{array}$ AACAGCAGTGACAGTGACAGCAGTGATAGCAGCAACAGCAGTGACAGCAGTGATAGCAGC

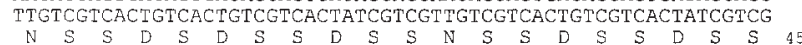
AACAGCAGTGATAGCAGTGAAAGCAGTAATAGTAGTGACAACAGCAATAGCAGTGACAGC TTGTCGTCACTATCGICACTTTCGTCATTATCATCACTGTTGTCGTTATCGTCACTGTCG $\begin{array}{lllllllllllllllllllll}\text { N } & S & S & D & S & S & E & S & S & N & S & S & D & N & S & N & S & S & D & S & 476\end{array}$ AGCAACAGCAGTGACAGCAGTGATAGCAGTGACAGCAGTAATAGTAGTGACAGCAGCAAT TCGTTGTCGTCACTGTCGTCACTATCGTCACTGTCGTCATTATCATCACTGTCGTCGTTA

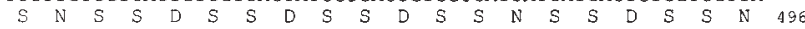
AGCAGTGACAGCAGCAACAGCAGTGACAGCAGTGATAGCAATAGCAGCGACAGCAGTGAC

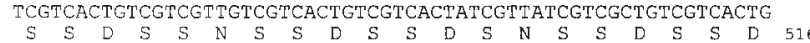
AGCAGCAACAGCAGCGATAGCAGTGACAGCAGTGATAGCAGTGACAGCAGTGACAGCAGT

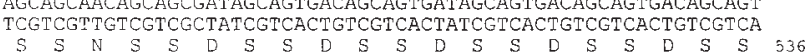
GATAGCAGCGACAGCAGT GATAGCAGTGACAGCAGT GACAGCAGTGATAGCAGTARTAGT

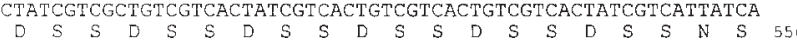
AGTGACAGCGGCAACAGCAGTGACAGCAGCGATAGCAGTGACAGCAGCGATAGCAGTGAC TCACTGTCGCCGTTGTCGTCACTGTCGTCGCTATCGTCACTGTCGTCGCTATCGTCACT AgCAGCGATAGCAGTGACAGCAGTGACAGCAGCAATAGCAGTGACAGCAGTGACAGCAGC TCGTCGCTATCGTCACTGTCGTCACTGTCGTCGTTATCGTCACTGTCGTCACTGTCGTCG GACAGCAGTGATAGCAGTGACAGCAGTGACAGCAGCGACAGCAGTGATAGCAGTGAAAGC CTGTCGTCACTATCGTCACTGTCGICACTGTCGTCGCTGTCGTCACTATCGTCACTTTCG D S S S D S S D S S D S S D S S D S S E S

AGTGATAGCAGCGATAGCAGTGACAGCAGCGACAGCAGTGACAGCAGTGACAGCAGTGAA TCACTATCGTCGCTATCGTCACTGTCGTCGCTGTCGTCACTGTCGTCACTGTCGTCACTT $\begin{array}{lllllllllllllllllllll}S & D & S & S & D & S & S & D & S & S & D & S & S & D & S & S & D & S & S & E & 636\end{array}$ AGCAGCAACAGCAGTGACAGCAGCGACAGCAGTGACAGCAGCGATAGCAGCGACAGCAGC

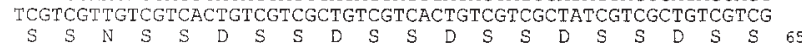
GACAGCAGCGATAGCAGTGACAGCAGCAATAGAAGTGATAGCAGCGACAGCAGTGATAGC

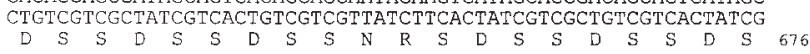
AGTGACAGCAGCGACAGCAGCGATAGCAGCGACAGCAGCGATAGTAGTGATAGCAGTGAC TCACTGTCGTCGCTGTCGTCGCTATCGTCGCTGTCGTCGCTATCATCACTATCGTCACTG

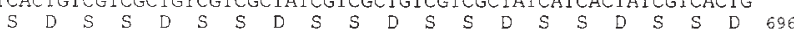
AGCAGTGACAGCAGCGACAGCAGTGACAGCAGCGACAGCAGTGACAGCAGCGACAGCAGT

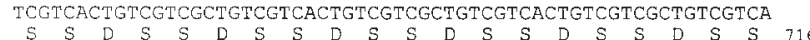
GACAGCAATGAAAGCAGCGACAGCAGT GACAGCAGCGATAGCAGTGACAGCAGCAACAGC

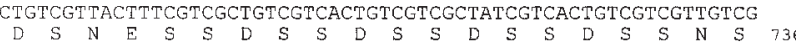
AGTGACAGCAGCGACAGCAGTGATAGCAGTGACAGCACATCTGACAGCAATGATGAGAGC

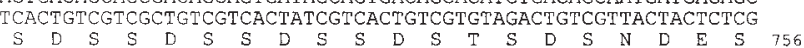
GACAGCCAGAGCAAGTCTGGTAACGGTAACAACAATGGAAGTGACAGTGACAGTGACAGT CTGTCGGTCTCGTTCAGACCATTGCCATTGTTGTTACCTTCACTGTCACTGTCACTGTCA GAAGGCAGTGACAGTAACCACTCAACCAGTGATGATTAG 3', CTTCCGTCACTGTCATTGGTGAGTTGGTCACTACTAATC $5^{\prime}$

E G S D S N H S T S D D

Fig. 2. Human DPP nucleotide sequence and deduced amino acid sequence. The human DPP DNA sequence was principally derived from two cDNA clones which overlap 215 bp (Fig. 1). The open reading frame spans 2364 bp encoding 788 amino acids with 461 serine and 205 aspartic residues. These residues are mostly arranged as 151 DSS and 32 DS. The deduced DPP amino acid sequence has an N-terminal sequence DDPN. $R G D$ at amino acid position 26, a putative cell attachment sequence, is bold and italicized. The conserved 19 C-terminal amino acids are underlined. (Accession number AF 09450).

(DDPNSSDESNG) (5) and to the human DPP Nterminal sequence, DDP (9).

Using primer C (Fig. 1), derived from the human 708 bp DNA sequence, and an oligo(dT)-adaptor primer to perform RT-PCR, we obtained a $2.4 \mathrm{~kb}$ PCR fragment (Fig. 1) which was subcloned and sequenced. These two clones overlapped by $215 \mathrm{bp}$. Additional PCR primers based upon the corrected human clones and the vector primers were synthesized to assist in sequencing the highly repetitive regions of DPP (Fig. 2).

The open reading frame of human DPP was found to span $2364 \mathrm{bp}$ encoding 788 amino acids (Fig. 2). It was rich in serine (461 residues, 58.6\%) and aspartic acid (205 residues, 26\%). These residues were found to be arranged as 151 $(\mathrm{DSS})_{\mathrm{n}}(n=1-16), 32$ (DS) and 37 (NSS) groups. The carboxyl-terminal 19 amino acids (Fig. 2) were 
Table 1.

Amino acid composition of deduced and native human DPP

\begin{tabular}{|c|c|c|c|c|}
\hline Amino acid & $\begin{array}{l}\text { Human }^{a} \\
\text { Res No. }\end{array}$ & $\underset{\%}{\operatorname{Human}^{\mathrm{a}}}$ & $\underset{\%}{\operatorname{Human}^{\mathrm{b}, \mathrm{c}}}$ & $\underset{\%}{\operatorname{Human}_{\%}^{\mathrm{b}, \mathrm{d}}}$ \\
\hline Asp & 205 & 26 & 36 & 41 \\
\hline Asn & 68 & 9 & & \\
\hline Ser & 461 & 58 & 38 & 42 \\
\hline Glu & 13 & 2 & 5 & 5 \\
\hline Gly & 16 & 2 & 5 & 4.6 \\
\hline Lys & 9 & 1.1 & 1.3 & 2.1 \\
\hline His & 1 & 0.1 & 1.4 & 0.4 \\
\hline Tyr & 1 & 0.1 & 0.44 & 0.4 \\
\hline Thr & 4 & 0.5 & 2 & 1.1 \\
\hline Pro & 1 & 0.1 & 5 & 0.6 \\
\hline Ala & 3 & 0.4 & 0.68 & 1.3 \\
\hline Cys & 1 & 0.1 & & 0 \\
\hline Ile & 0 & 0 & 0 & 0.3 \\
\hline
\end{tabular}

${ }^{a}$ Amino acid composition derived from deduced protein sequence; \%, amino acid composition represented in percent.

$\mathrm{b}$ Amino acid composition derived from native protein.

c TAKAGI \& VeIs (15).

${ }^{\mathrm{d}}$ MCCuRdy et al. (28).

identical to those published earlier for rat $(5,14)$ and mouse (6) DPP. Subsequently, the complete open reading frame (Fig. 2) was cloned using an upstream and a downstream primer specific to this human gene. Partial sequencing confirmed the molecular cloning of a human DPP. In aggregate, these data strongly support designation of this molecule as a human DPP.

According to the present data, this human DPP has 299 (788-489) more amino acids than mouse DPP (6), 548 (788-240) more than rat DPP as reported by Ritchie \& WANG (5), and 573 (788-215) more than the DPP region of rat DMP-3 (14). Like mouse (6) but unlike rat (5), human DPP contains an RGD (Arg-Gly-Asp) sequence, starting at position 26 (Fig. 2).

Hydropathy distribution analysis revealed that the protein is extremely hydrophilic (data not shown). The net charge of the core protein (before phosphorylation) was calculated to be -206 with an isoelectric point of 2.65. The molecular weight for the core protein is $76,906 \mathrm{Da}$. The deduced amino acid percentages obtained from our cDNA and those obtained from native proteins by conventional amino acid analysis $(10,15)$ are compared in Table 1. Our human DPP preparation as reported (9) was likely contaminated by collagen. Increased purity of such preparations has been associated with a relative increase in aspartic acid and serine residues, concomitant with a decrease in glycine and proline (Chang, unpublished data). These observations and the possibility of multiple DPP molecules (1) could account for the apparent discrepancies between the deduced amino acid composition data and those obtained from extracted DPP (Table 1).

DPP is rich in phosphoserine (1), and serine residues are likely to be phosphorylated posttranslationally by protein kinase(s). Therefore, we examined the potential phosphorylation sites of our deduced human DPP sequence and found consensus sequences for casein kinase I [Asp/Glu/ pSer-X-X-Ser/Thr (16-18)] and casein kinase II [Ser/Thr-X-X-Asp/Glu/pSer (16, 19-21)]. Overall, $53 \%$ of the serine residues could potentially be phosphorylated by casein kinases I or II. With this number of phosphoserines, DPP could carry an additional charge of -395 from phosphate groups alone $[-1.6 / \operatorname{Ser}(\mathrm{P})$ or $\operatorname{Thr}(\mathrm{P})]$. Therefore, DPP theoretically could carry an overall net charge of -601 at physiological $\mathrm{pH}$. In addition, using Asn-XSer/Thr as consensus $\mathrm{N}$-glycosylation sites $(22,23)$, we found 47 potential sites which, if glycoslyated, might further increase the net charge and the molecular mass.

It is widely accepted that DPP has an essential role in the regulation of dentin mineralization by virtue of its amino acid composition and repetitive arrangement of aspartic acid (D) and serine (S) residues $(1,3,24)$. The presence of a similar composition and arrangement of $\mathrm{D}$ and $\mathrm{S}$ residues in human DPP (Fig. 2) strongly supports a putative role for this protein in human dentin mineralization. In addition, the presence of a cell-binding RGD sequence near the N-terminus in mouse (6) and human deduced DPP (Fig. 2) sequences suggests that human DPP may attach cells expressing the appropriate integrins. Since DPP is 
expressed in pre-ameloblasts during odontogenesis (25), this might possibly be related to the cellular differentiation processes occurring at this stage. In contrast, however, no RGD sequence was found in the deduced rat DPP sequence $(5,8)$, and experiments are clearly needed to investigate the function of this RGD sequence in human and mouse DPP.

Preliminary data (K. Gu, unpublished) imply that the human DPP mRNA reported here is a $3^{\prime}$ extension of an open reading frame encoding for human DSP and the DSP-PP linker region, and suggest that in humans, like rats and mice, a single gene encodes DSP and DPP. Additionally, DPP isoforms $(1,26,27)$ as well as multiple DPP transcripts have been identified in rat $(1,3,8)$. Our preliminary data also imply the presence of multiple human DPP molecules (K. Gu, unpublished).

Current experiments are focusing on sequencing the human DSP region, isolating and characterizing DSP from human dentin, and determining the patterns of expression of these molecules during tooth formation and reparative dentinogenesis.

\section{References}

1. Butler WT. Dentin matrix proteins. Eur J Oral Sci 1998; 106: 204-210.

2. Butler WT, Bhown M, Dimuzio MT, Linde A. Nonocollagenous proteins of dentin. Isolation and partial characterization of rat dentin proteins and proteoglycans using a three- step preparative method. Collagen Rel Res 1981; 1: 187-199.

3. Ritchie HH, Ritchie DG, Wang LH. Six decades of dentinogenesis research. Historical and prospective views on phosphophoryn and dentin sialoprotein. Eur J Oral Sci 1998; 106: 211-220.

4. Ritchie HH, WANG L-H. A mammalian bicistronic transcript encoding two dentin-specific proteins. Biochem Biophys Res Commun 1997; 231: 425-428.

5. Ritchie HH, Wang L-H. Sequence determination of an extremely acidic rat dentin phosphoprotein. $J$ Biol Chem 1996; 271: 21,695-21,698.

6. Macdougall M, Simmons D, Luan X, Nydegger J, Feng J, Gu T. Dentin phosphoprotein and dentin sialoprotein are cleavage products expressed from a single transcript coded by a gene on human chromosome 4. J Biol Chem 1997; 272: $835-842$

7. Feng JQ, Luan X, Wallace J, Jing D, Ohshima T, Kulkarni AB, D'souza RN, Kozak CA, Macdougall M. Genomic organization, chromosomal mapping, and promoter analysis of the mouse dentin sialophosphoprotein (Dspp) gene, which codes for both dentin sialoprotein and dentin phosphoprotein. J Biol Chem 1998; 273: 9457-9464.

8. George A, Srinivasan R, Thotakura S, Veis A. The phosphophoryn gene family: identical domain structures at the carboxyl end. Eur J Oral Sci 1998; 106: 221-226.

9. Chang SR, Chiego D, Clarkson BH. Characterization and identification of a human dentin phosphophoryn. Calcif Tissue Int 1996; 59: 149-153.

10. McCurdy SP, Clarkson BH, Feagin FF. Comparison of phosphoprotein isolated from mature and immature human tooth roots. Arch Oral Biol 1992; 37: 1057-1065.
11. Macdougall M. Refined mapping of the human dentin sialophosphoprotein (DSPP) gene within the critical dentinogenesis imperfecta type II and dentin dysplasia type II loci. Eur J Oral Sci 1998; 106: 227-323.

12. Takagi Y, Veis A. Matrix protein differences between human normal and dentinogenesis imperfecta dentin, VEIS A. The chemistry and biology of mineralized connective tissue, Vol. II. New York: Elsevier North-Holland, 1981; 223-243.

13. Takagi Y, Veis A, Sauk JJ. Relation of mineralization defects in collagen matrices to non-collagenous protein components: identification of a molecular defect in dentinogenesis imperfecta. Clin Orthop 1983; 176: 282290.

14. George A, Bannon L, Sabsay B, Dillon JW, Malone J, Veis A, Jenkins NA, Gilbert DJ, Copeland NG. The carboxyl-terminal domain of phosphophoryn contains unique extended triplet amino acid repeat sequences forming ordered carboxyl-phosphate interaction ridges that may be essential in the biomineralization process. J Biol Chem 1996; 271: 32,869-32,873.

15. Takagi Y, Veis A. Isolation of phosphophoryn from human dentin organic matrix. Calcif Tissue Int 1984; 36: 259-265.

16. Pinna L. Casein kinase 2: an "eminence grise" in cellular regulation. Biochem Biopys Acta 1990; 1054: 267-284.

17. Flotow H, Graves P, Wang A, Fiol C, Roeske R, Roach P. Phosphate groups as substrate determinants for casein kinase I action. J Biol Chem 1990; 265: 14,264-14,269.

18. Flotow H, Roach P. Role of acidic residues as substrates determinants for casein kinase I. J Biol Chem 1991; 266: 3724-3727.

19. Meggio F, Marchiori F, Borin G, Chessa G, Pinna L. Synthetic peptides including acidic clusters as substrates and inhibitors of rat liver casein kinase TS (type-2). J Biol Chem 1984; 259: 14,576-14,579.

20. Kuenzel E, Mulligan J, Summercorn J, Krebs E. Substrate specificity determinants for casein kinase II as deducted from studies with synthetic peptides. J Biol Chem 1987; 262: 9136-9140.

21. Meggio F, Perich J, Meyer H, Hoffmann-Posorske E, Lennon D, Johns R, Pinna L. Synthetic fragments of betacasein as model substrates for liver and mammary gland casein kinases. Eur J Biochem 1989; 186: 459-464.

22. Marshall RD. Glycoproteins. Ann Rev Biochem 1972; 41: 673-702.

23. BAuSE E. Structural requirements on $\mathrm{N}$-glycosylation of proteins. Studies with proline peptides as conformational probes. Biochem J 1983; 209: 331-336.

24. Veis A, Wei K, Sfeir C, George A, Malone J. Properties of the (DSS)n triplet repeat domain of rat dentin phosphophoryn. Eur J Oral Sci 1998; 106: 234-238.

25. Bègue-Kirn C, Ruch J-V, Ridall AL, Butler WT. Comparative analysis of mouse DSP and DPP expression in odontoblasts, preameloblasts, and experimentally induced odontoblast-like cells. Eur J Oral Sci 1998; 106: 254-259.

26. Linde A, Bhown M, Butler WT. Noncollagenous proteins of dentin. A re-examination of proteins from rat incisor dentin utilizing techniques to avoid artifacts. $J$ Biol Chem 1980; 255: 5931-5942.

27. Butler WT, Bhown M, Dimuzio MT, Cothran WC, Linde A. Multiple forms of rat dentin phosphoproteins. Arch Biochem Biophys 1983; 225: 178-186.

28. McCurdy SP, Clarkson BH, Speirs RL, Feagin FF. Phosphoprotein extraction from the dentine/cementum complex of human tooth roots. Arch Oral Biol 1990; 35: 347-357. 